\title{
Knowledge, Attitude, and Practice (KAP) towards Family Planning among Women of Reproductive Age in Fiji
}

\author{
Jay Lincoln ${ }^{1}$, Masoud Mohammadnezhad ${ }^{1} \&$ Sabiha Khan ${ }^{1}$ \\ ${ }^{1}$ School of Public Health and Primary Care, Fiji National University, Suva, Fiji Islands \\ Correspondence: Masoud Mohammadnezhad, Associate Professor in Public Health (Health Promotion), School of \\ Public Health and Primary Care, Fiji National University, Suva, Fiji. Tel: 679-972-6127
}

Received: January 10, 2021 Accepted: February 15, 2021 Online Published: February 22, 2021

doi:10.5539/gjhs.v13n4p24 URL: https://doi.org/10.5539/gjhs.v13n4p24

\begin{abstract}
Background: Globally, family planning is one of the most important public health issues in both developed and developing countries due to high unmet needs.

Objective: To assess the level of knowledge, attitudes and practice (KAP) towards family planning among women of reproductive age in Suva, Fiji.

Methods: The study applies a cross-sectional quantitative method using a self-administered questionnaire to collect data. Using random sampling, a sample of 325 women was used for the study and they were sought from three health clinics in Suva, Fiji. The questionnaire was administered to the respondents who met the inclusion criteria and they provided information that mostly involved rating the different issues and identifying their level of KAP. This data was analyzed using the statistics program SPSS version 25.
\end{abstract}

Results: The mean age of the participants was $31.53( \pm 7.35)$ years. A majority of the participants $(64.6 \%)$ belonged to a Christian church while $65.2 \%$ belonged to iTaukei ethnic group. Approximately half of them (45.6\%) had a high level of knowledge while $53.5 \%$ had moderate knowledge with only $0.9 \%$ reporting that they had poor knowledge. In terms of attitudes, $54.2 \%$ of the participants had high level or positive attitudes towards family planning with only a small proportion of $0.3 \%$ had low level or poor attitudes. In terms of practice, a majority of the participants $(65.9 \%)$ had medium level of practice towards family planning while $24.6 \%$ showed a poor level with $9.5 \%$ having a high or good level of practice towards family planning.

Conclusions: This study identified that women had medium level of knowledge and practice towards family planning, but their attitude was relatively high. Using behavioral change models such as health belief model (HBM) that focus on perceived benefits and barriers may help to promote KAP towards family planning among Fijian women.

Keywords: contraception, birth control, knowledge, attitude, practice, family planning, Fiji

\section{Introduction}

Family planning refers to the use of different methods and strategies for enabling men and women to make informed decisions about child bearing. It consists of the methods that men and women use to space their pregnancies and control the numbers of off springs that they want to have (Brown et al., 2014). Understanding the levels of contraceptives use in the community is essential in understanding changes in fertility and the reproductive health across regions worldwide. Studies such as the UN world report indicate that $64 \%$ of all women in unions or marriages globally use contraceptives (United Nations, 2015). However, the level of such usage is relatively low in developing nations such as the Oceania region where the rate was 40\% in 2015 (Alkema et al., 2006). There are significant regional differences with $12 \%$ of women in civil unions or marriages globally having an unmet need for family planning (McMurray \& Lucas, 1990). This rate of unmet contraceptive needs was high at $22 \%$ in least developed countries (Kennedy et al., 2011). The women with unmet contraceptive needs are those who want to stop or delay childbirth but are not using any method of contraception. The prevalence of contraceptive use is expected to grow towards 2030 with the highest rates being expected in Oceania and Sub Saharan Africa (Alkema et al., 2006; Cates et al., 2010).

Low motivation for the use of family planning in different settings is cited as resulting from lack of adequate information about contraceptives, lack of accessibility, fear of side effects, and negative attitudes due to social and 
religious sentiments (Dehlendorf et al., 2010). The quality of education available on the subject can be associated with provider bias and most of the challenges are associated with lack of awareness (Naidu et al., 2017).

In Fiji, reproductive health services are delivered through all health centers in the Central, Eastern, Northern and Western divisions. These services are free of charge for Fijian population (Ministry of Health, Fiji, 2016). According to Gyaneshwar, et al., (2016), Fiji's population growth rate declined to $0.83 \%$ in 2010 from between $1.39 \%$ to $1.41 \%$ in the period $2000-2009$. However, the decline is not attributed to family planning but to other factors such as the movement of women away from the home to non-familial work in the labor market (Kuang et al., 2016).

In Fiji, one of the key issues that have been identified as a barrier to the uptake of contraception is the lack of female providers. This was cited as a key issue for a third of the women in Fiji and New Zealand (Cammock et al., 2017). The issue of cost was also identified as a major barrier contributing to unmet needs for contraception (Bradley et al., 2012). The role of KAP in using contraception is always questionable. As there is no study conducted yet in Fiji among women regarding the family planning it important to measure the level of KAP. Therefore, this study focuses on the issues and challenges facing Fiji in terms of low uptake of family planning to evaluate the level of knowledge about the concept of family planning, its methods, health aspects and barriers, the level of attitudes toward family planning approaches, use of contraceptive approaches and barriers and the level of family planning practices and use of contraceptive methods among women of reproductive age in Suva, Fiji in 2017.

\section{Methods}

This quantitative, cross - sectional study was conducted among women who attended three designated health clinics around Suva, Fiji from 15 March to 28 April 2017. The study population included all Fijian women of reproductive age. The inclusion criteria included women, ages 15-49 who were married (target group for family planning services) and were attended any one of the three identified family planning clinics under study from 15 March to 28 April 2017. Furthermore, the study participant must have been a Fijian - anybody in Fiji who possesses Fijian citizenship. The exclusion criteria included women under the age of 15 or age 50 and over and those women who are not happy to participate in the study. Participants who met the inclusion and exclusion criteria who consented to participate in the study were asked to fill a questionnaire survey to identify the level of KAP towards family planning. The sampling was conducted randomly and proportional sampling was applied to determine the sample size by considering a 50\% prevalence of KAP about family planning, $95 \%$ confidence interval, 5\% margin error, and 5\% non-respondent rate which gives a total sample size of 342 .

A self -administered questionnaire was used to collect data (Table 1), which was tested in a pilot study for validity. Face validity was conducted among 10 women who met the study inclusion criteria and content validity was done by three experts in the field of family planning. Based on the geographical distribution and covering a large number of population, three randomly selected family planning health clinics were chosen including; Nuffield Clinic in Tamavua, Wellness Center for Women/Oxfam) at the Colonial War Memorial Hospital (CWMH) in Suva, and Samabula Health Center in Suva. These health centers provide reproductive health services such as; safe motherhood, infant and child care, adolescent health care, family planning and prevention of abortion, STI-HIV prevention and management, and basic infertility services and management of gynaecological morbidity including reproductive tract cancers \& infections (Ministry of Health Fiji, 2016).

Table 1. Questions were tested for the KAP

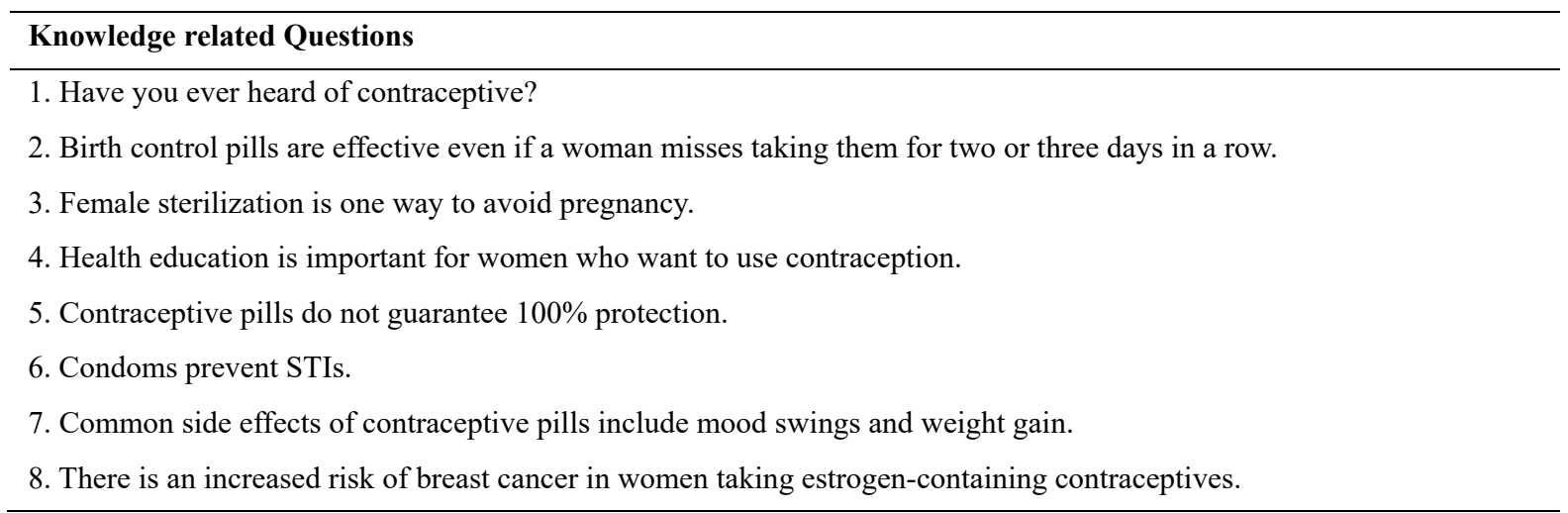


9. Women using the birth control shot must get an injection every three months.

10. If a woman is having side effects of one kind of contraceptive pill, switching to another type might help.

11. Using both a condom and the pill is considered to be a very effective contraceptive.

12. Using the pill increases a woman's risk of ovarian, endometrial or cervical cancer.

\section{Attitude related Questions}

1. Contraceptives should be used to limit my number of children.

2. Contraceptives should be used to increase the time interval between my childbirths.

3. Spacing will allow a child to be healthier.

4. The ideal age of having a first child is 20-30.

5. The ideal number of children should be between 3-5

6. Contraceptives provide a sense of safety.

7. The method of contraception I am using is adequate.

8. Contraceptives benefit males too.

9. Discussion about contraception with spouse is embarrassing.

10. My husband does not approve my use of contraceptives.

11. Contraceptive methods can protect the health of family and community.

12. Religious beliefs can prevent women from using contraceptives.

13. Cultural beliefs can prevent women from using contraceptives.

14. Husband's objections to contraceptives can prevent women from using contraceptives.

15. Change in male attitudes on contraceptives may improve contraceptive use.

\section{Practice related Questions}

1. How many times a year do you visit a health center for family planning services?

2. Do you use contraceptives to prevent unplanned pregnancy?

3. Have you ever had any unplanned pregnancy due to lack of contraceptive use?

4. Do you use contraceptives every time when you do not intend to get pregnant?

5. I use different types of contraceptives.

6. My current method of contraceptive changes from time to time.

7. Do you practice any traditional contraceptive methods including withdrawal, infertility period, herbal and breast feeding if you were not using any contraceptives?

There were 12 knowledge related questions that carried a total of 24 correct responses. Each correct response was given a score of 2 and a wrong response a score of 0 whereas a don't know response carried a score of 1 . Total points to be scored were 24 and the minimum was 0 . On assessment, a modified Bloom's cut off points were used where a score of $80-100 \%$ of correct responses meant a good knowledge, a score of 50-79\% was a level of medium level knowledge and a poor knowledge was for the respondents with a score less than $50 \%$ of the correct responses. Modified Bloom cut off points were adopted from John's knowledge, attitude and practice study (John, 2011; Yimer et al., 2013). The scores, with their respective knowledge levels were (a) 0 - 11 as "Poor" (low level) knowledge (b) 12- 18 as "Moderate" (medium level) knowledge and (c) $19-24$ as "Good" (high level) knowledge.

The participants' attitudes was assessed using 15 questions with Likert scale. The questions on a Likert scale were ranged from high level to low level responses such as 5 as "Strongly Agree", 4 as "Agree", 3 as "Neutral", 2 as "Disagree" and 1 as "Strongly Disagree". The total score obtained for respondents was a maximum of 75 with a minimum of 15. Combined scores of less than 35 were categorized as low level attitude, from 35 to 56 as medium level attitude, and 57 and above as high level attitude. The mean score was calculated and those scoring above the mean were classified as high level attitude and scores below the mean was classified as low level attitude towards family planning whereas those scores within between low and high level attitude were classified as medium level 
attitude.

The participants' practice was assessed by 7 questions about family planning in a scale of $1-5$ with 1 as Never, 2 as Seldom, 3 as Sometimes, 4 as Usually and 5 as Always. The maximum score was 35 whereas the minimum score was 7. Therefore, 7-15 was scored as Poor (low level) practice; 16- 24 were scored as Moderate (medium level) practice and 25 - 35 was scored as Good (high level) practice (Tavoosi, 2004; Mohammadnezhad, 2015; Tekanene et al., 2017). The data was analyzed with SPSS Version 22 statistical software. The frequency and percentage of KAP are presented in tables and graph.

Data was collected from the selected study sites by the main researcher after the required ethical approvals of the study had been secured from the College of Health Research Ethics Committee (CHREC) and MHMS Fiji National Heath Research and Ethics Review Committee (FNHRERC). All participants were informed about the objectives of study using an information sheet and were asked to sign a consent form before participating in this study. They were informed that participating in this study is totally voluntary and their information will be confidential. Participants were given 15-20 minutes to fill the questionnaire. If they needed support to complete the questionnaire, the main researcher assists them. To ensure the questionnaire is filled completely, all the questionnaires were checked by the main researcher before collecting from the participants.

\section{Results}

The focus of the study, which was conducted on a sample of 325 women visiting the three designated health clinics for this study, was to evaluate the KAP that women have towards family planning. The average age of the participants was $31.53( \pm 7.35)$ years and most of the participants were in the age bracket of 25-29 years accounting for $26.8 \%$ of the sample. About two-thirds (65.2\%) of the participants were from the iTaukei ethnic group. Majority of the participants were Christians accounting for $64.6 \%$, which were 210 respondents. Another interesting element of the sample was that it consisted mostly of women that were involved in non-familial work as active participants in the labor market where $60.6 \%$ were employed compared to $39.4 \%$ that were housewives. The sample was mostly based in urban areas where $70.5 \%$ were residents in urban centers. In terms of the number of children, $63.1 \%$ has $1-3$ children indicating the declining fertility rates in the country caused by higher education levels in the community coupled with the involvement of the women in the labor market. The income level for $34.2 \%$ of the women was $\$ 8,000.00-\$ 14,999.00$ (Table 2).

Table 2 Demographic characteristics of participants $(\mathrm{n}=325)$

\begin{tabular}{llll}
\hline Variables & Categories & Frequency (n) & Percentage (\%) \\
\hline \multirow{4}{*}{ Age } & $15-19 \mathrm{yrs}$ & 5 & 1.5 \\
& $20-24 \mathrm{yrs}$ & 48 & 14.7 \\
& $25-29 \mathrm{yrs}$ & 87 & 26.8 \\
& $30-34 \mathrm{yrs}$ & 72 & 22.2 \\
& $35-39 \mathrm{yrs}$ & 55 & 16.9 \\
& $40-45 \mathrm{yrs}$ & 37 & 11.4 \\
& $45-49 \mathrm{yrs}$ & 21 & 6.5 \\
\hline \multirow{3}{*}{ Religion } & Christian & 210 & 64.62 \\
& Hindu & 69 & 21.23 \\
& Muslim & 16 & 4.92 \\
Ethnicity & Others & 30 & 9.23 \\
\hline \multirow{2}{*}{ Employment } & iTaukei & 212 & 65.2 \\
& Indian & 88 & 27.1 \\
\hline
\end{tabular}




\begin{tabular}{llll}
\hline & $<7,999$ & 90 & 27.7 \\
Annual income (FJD) & $8,000-14,999$ & 111 & 34.2 \\
& $15,000-24,999$ & 109 & 33.5 \\
& $25,000-35,000$ & 15 & 4.6 \\
\hline \multirow{2}{*}{ Number of children } & 0 & 36 & 11.08 \\
& $1-3$ & 205 & 63.08 \\
Area of residence & $4-6$ & 82 & 25.23 \\
\hline
\end{tabular}

The defiles of participants answer to KAP questions is presented in previous publication (Lincoln et al., 2018). In terms of knowledge, $53.5 \%$ had moderate level knowledge while $45.6 \%$ had a high level knowledge with only $0.9 \%$ having low level knowledge of family planning. Most of the participants had positive or high level of attitudes towards family planning as shown by the high level recorded by $54.2 \%$ of the sample while $45.5 \%$ indicated a moderate level. Only $0.3 \%$ had low level of attitudes towards family planning. In terms of practice, most participants were found to have a moderate level of practice towards family planning. This was almost two-thirds of the sample (65.9\%) while $24.6 \%$ had a low level. Only $9.5 \%$ had a high level of practice in relation to using family planning (Table 3 ).

Table 3. Level of KAP towards family planning

\begin{tabular}{llc}
\hline Variables & Frequency (n) & Percentage (\%) \\
\hline Knowledge & 148 & 45.6 \\
Good (High Level) Knowledge ( $>19)$ & 174 & 53.5 \\
Moderate (Medium Level) Knowledge (12-18) & 3 & 0.9 \\
Poor (Low Level) Knowledge (<11) & 176 & 54.2 \\
\hline Attitude & 148 & 45.5 \\
Good (High Level) Attitude ( $>57)$ & 1 & 0.3 \\
Moderate (Medium Level) Attitude (36 - 56) & & 9.5 \\
Poor (Low Level) Attitude (15-35) & 31 & 65.9 \\
\hline Practice & 214 & 24.6 \\
Good (High Level) Practice (25 - 35) & 80 & \\
Moderate (Medium Level) Practice (16-24) & & \\
Poor (Low Level) Practice (7 - 15) & & \\
\hline
\end{tabular}

\section{Discussion}

The focus of the research was to identify the level of KAP of Fijian women towards family planning. These results of the study revealed that the participants have relatively medium level of knowledge and practice towards family planning and high level of attitudes towards family planning. The results of the analysis indicate that health education and family planning initiatives that have been undertaken in the country have not improved the usage of family planning in Fiji as evidenced by the chronic low contraceptive prevalence rate (CPR) in the 40s over the last three decades (Kennedy, 2013). Yet despite the lack of improvement of the overall CPR in Fiji to date, the results indicate that the initiative implemented by the government have played a some role in improving women's knowledge towards the availability of modern family planning methods as evidenced by the KAP results noted in table 2. The results are in line with the findings of studies such as Gyaneshwar et al., (2017), which have indicated that government initiatives have produced beneficial outcomes in reducing the crude birth rate in Fiji to sustainable 
levels.

An interesting element of the findings is that most people are knowledgeable about family planning. There are regional differences in practice but these are mainly affected by the cultural and social practices of the community. This view is supported by the findings of Alkema, et al., (2006). In Fiji, a key challenge cited as preventing some women from seeking family planning services is that they lack female providers. The cultural practices bar them from going to male doctors in search of contraceptive services as found out in Cammock et al., (2017). While awareness regarding contraceptives is available, there are some challenges in relation to its application and the depth of how much the women know. One interesting element of knowledge was that only $54 \%$ of the respondents acknowledged that missing contraceptives for 2-3 days consecutively could make them ineffective. It would be better for the community if the women had better knowledge of contraceptives, because this would directly affect their practice. The fact that the respondents with high knowledge about contraceptives made up $45 \%$ of the sample means that there is a lot of room for improving awareness / attitudes levels.

It is clear that most of the respondents identified themselves as Christians, which makes up $64.6 \%$ of the sample. This may have a significant influence on the practice and attitudes that the women reported towards family planning. The basis of the Christian beliefs in Fiji about contraception as being unacceptable can be associated with the colonial foundations implemented by the missionaries (Gyaneshwar et al., 2016; Hodges, 2008). The issue of contraception uptake has also been associated with gender disparities and the views of men that are in some cases a barrier for women in using contraception (Vasilenko et al., 2015; Sewak \& Singh, 2012). As indicated in a study by Delaibatiki-Cammock (2015), the issue of male domination of reproductive health decisions is a significant barrier to the uptake of contraceptives in Fiji (Delaibatiki-Cammock 2015). This issue is highly apparent in the Indo-Fijian community, but has also been highlighted as a major factor in many developing nations and regions (Chandra \& Lewai, 2005). Another major issue that needs consideration in understanding family planning in Fiji is that there are significant differences in the rates of usage as well as awareness across regions and demographics. For example, Mustafa et al., (2008) indicated that the issue of contraceptives use differs considerably between rural and urban populations. There is lower awareness in rural populations in many developing nations. This element means that the findings of the current study have to be interpreted with caution (Naidu et al., 2017). While the population of Fiji is mostly rural, the sample only had about $29 \%$ of rural respondents. This means the findings may be skewed towards the awareness levels, practice and attitudes of urban women.

These findings indicate the need for more educational strategies for the community on family planning. Social marketing has been cited as a significant strategy for ensuring that the needs of the community for contraceptives are effectively addressed (Mustafa et al., 2008; Cleland et al., 2006). It also shows that the Fiji government needs to consider ways of enhancing the uptake of contraceptives by moving beyond providing awareness (Cleland et al., 2014). This can be achieved by including men in awareness and educational programs on family planning. Addressing cultural and social barriers would also play a major role in reducing the level of unmet family planning needs (Bongaarts, 2014).

The findings of this study highlight the urgency of implementing policies that would seek to educate women about proper family planning methods and techniques specially targeted at women with poorer socioeconomic backgrounds

\subsection{Limitation of the Study}

Although, this study is evidenced by the fact that it is one of very few studies concerned with the state of KAP of family planning in Fiji involving women of all races and social status and as such it provides a useful source of empirical information and as such this study is relevant primarily for public health policy makers in this country, there were few limitations such as; lack of generalizing the results to all pregnant women as that usually happens in cross-sectional studies. The results can be affected as the data collected used a self - report questionnaire. Lastly, the lack of causality from the results could have been attributed to the type of study used in this study

\section{Conclusions}

From the results of this study, it is concluded that the quantitative findings showed that women of reproductive age (15-49) had a fair (moderate) level of knowledge towards family planning. From the findings of this study, it is clear that the level of knowledge of family planning in Fiji is moderate which leaves much room for improvement. The majority of the sampled women have medium level of knowledge and practice towards family planning, but their attitude considerations are relatively high. It is important for the government to develop ways of responding to the cultural, social, and religious barriers to the uptake of family planning by Fijian women. Gender disparities 
require further research to identify how they influence the perceptions and practices.

\section{Ethical Considerations}

Data collection was done after the researcher received ethical approval from the College of Health Research Ethics Committee (CHREC) of Fiji National University and the Ministry of Health's Fiji National Heath Research Ethics Review Committee (FNHRERC).

\section{Acknowledgments}

We thank all the nurses at Nuffield Family Health Unit, Samabula Health Center Family Health Unit Clinic and Oxfam/Women's Wellness Center for their tireless efforts and tremendous assistance they provided to us in ensuring the process of data gathering went smoothly. We also appreciate all the women who dedicated a small yet precious portion of their busy schedules to participate in the survey for this study.

\section{Funding}

This research did not receive any specific grant from funding agencies in the public, commercial, or not-for-profit sectors.

\section{Competing Interests Statement}

The authors declare that there are no competing or potential conflicts of interest.

\section{References}

Alkema, L., Kantorova, V., \& Menozzi, C. (2006). National, regional, and global rates and trends in contraceptive prevalence and unmet need for family planning between 1990 and 2015: A systematic and comprehensive analysis. The Lancet, 381(9878), 1642-1652. https://doi.org/10.1016/S0140-6736(12)62204-1.

Bradley, S. K., Croft, T. N., Fishel, J. D., \& Westoff, C. F. (2012). Revising unmet need for family planning. Calverton: ICF International.

Brown, W., Druce, D., \& Bunting, J. (2014). Developing the " 120 by 20 " goal for the Global FP2020 Initiative. Studies in Family Planning, 45, 73-84. https://doi.org/10.1111/j.1728-4465.2014.00377.x

Bongaarts, J. (2014). The impact of family planning programs on unmet need and demand for contraception. Studies in Family Planning, 45(2), 247-262. https://doi.org/10.1111/j.1728-4465.2014.00387.x.

Cammock, R., Herbison, P., Lovell, S., \& Priest, P. (2017). Family planning unmet need and access among iTaukei women in New Zealand and Fiji. New Zealand Medical journal, 130(1462), 46-53.

Cates, W., Stantback, J., \& Maggwa, B. (2010). Global development: Family planning and the Millennium Development Goals. Science, 329(5999), 1603. https://doi.org/10.1126/science.1197080.

Chandra, D., \& Lewai, V. (2005). Women and men of Fiji Islands: Gender statistics and trends. Suva: Population Studies Programme, University of the South Pacific.

Cleland, J., Bernstein, S., \& Ezeh, A. (2006). Family planning: the unfinished agenda. The Lancet, 68(9549), 810-1827. https://doi.org/10.1016/S0140-6736(06)69480-4

Cleland, J., Harbison, S., \& Shah, I. H. (2014). Unmet need for contraception: issues and challenges. Studies in Family Planning, 45(2), 105-122. https://doi.org/10.1111/j.1728-4465.2014.00380.x.

Dehlendorf, C., Rodriguez, M. I., \& Levy, K. (2010). Disparities in family planning. American Journal of Obstetrics and Gynecology, 202(3), 214-220. https://doi.org/10.1016/j.ajog.2009.08.022.

Delaibatiki-Cammock, R. (2015). Conference Abstracts. 'iTaukei family planning behavior in Fiji's central division'. Pacific Journal of Reproductive Health, 1, 34-46.

Gyaneshwar, R., Naidu, S. L., Naidu, S., \& Baseisei, M. (2016). A historical context of family planning in Fiji and some thoughts for future directions. Fiji Journal of Public Health.

Hodges, S. (2008). Contraception, colonialism and commerce: Birth control in South India, 1920-1940. Aldershot, England: Ashgate.

John, J. (2011). The knowledge, attitude, practice and perceived barriers towards screening for premalignant cervical lesions among women aged 18 years and above in Songea urban, Ruvuma. Muhimbili University of Health and Allied Sciences. Retrieved from http://ihi.eprints.org/962/1/muhas

Kennedy, E., Gray, N., Azzopardi, P., \& Creati, M. (2011). Adolescent fertility and family planning in East Asia and the Pacific: a review of DHS reports. Reproductive Health, 8, 11. https://doi.org/10.1186/1742-4755-8-11 
Kennedy, E. C., Mackesy-Buckley, Subramaniam, S., Demmke, A., Latu, R., Robertson, A. S., \& Luchters, S. (2013). The case for investing in family planning in the Pacific: Costs and benefits of reducing unmet need for contraception in Vanuatu and the Solomon Islands. Reproductive Health, 10(30). https://doi.org/10.1186/1742-4755-10-30.

Kuang, B., \& Brodsky, I. (2016). Global Trends in Family Planning Programs, 1999-2014. International Perspectives on Sexual and Reproductive Health, 42(1), 33 - 44. https://doi.org/10.1363/42e0316

Lincoln, J., Mohammadnezhad, M., \& Khan, S. (2018). Knowledge, Attitudes, and Practices of Family Planning Among Women of Reproductive Age in Suva, Fiji in 2017. J Women's Health Care, 7, 431. https://doi:10.4172/2167-0420.1000431

McMurray, C., \& Lucas, D. (1990). Fertility and Family Planning in the South Pacific Islands. Australia Working Paper No 90/10. Canberra: National Centre for Development Studies, The Australian National University.

Ministry of Health. (2016). Annual reports. Ministry of Health, Republic of the Fiji Islands

Mohammadnezhad, M., Wilson, C., Ratcliffe, J., Tsourtos, G., Ullah, Sh., \& Ward, P. (2015). Knowledge and Attitude Towards Smoking-related Cancers Among Older Greek-Australian: A Mixed- Methods Study. Journal of Family Medicine, 2(6), 1043.

Mustafa, R., Afreen, U., \& Hashmi, H. A. (2008). Contraceptive knowledge, attitude and practice among rural women. Journal of the College of Physicians and Surgeons Pakistan, 18(9), 542-545.

Naidu, S. L., Heller, G. Z., Koroi, Z., Dearkin, L., \& Gyaneshwar, R. (2017). Knowledge, attitude, practice and barriers regarding safe sex and contraceptive use in rural women in Fiji. Pacific Journal of Reproductive Health, 1(5), 223-231. https://doi.org/10.18313/pjrh.2017.901.

Sewak, A., \& Singh, G. (2012). Assessment of the Fiji-based condom social marketing (CSM) program. Sexuality \& Culture, 16(4), 389-407. https://doi.org/10.1007/s12119-012-9128-3

Tavoosi, A., Zaferani, A., Enzevaei, A., Tajik, P., \& Ahmadinezhad, Z. (2004). Knowledge and attitude towards HIV/AIDS among Iranian students. BMC Public Health, 4, 17

Tekanene, M. U., Mohammadnezhad, M., \& Khan, S. (2017). Knowledge, Attitude and Practice (KAP) study towards Type 2 Diabetes Mellitus (T2DM) among adults who attend public health clinics in South Tarawa, Kiribati in 2017 (Masters thesis. School of Public Health and Primary Care. Fiji National University).

United Nations. (2015). Trends in Contraceptive Use Worldwide. New York: UN Department of Economic and Social Affairs, Population Division.

Vasilenko, S. A., Kreager, D. A., \& Lefkowitz, E. S. (2015). Gender, contraceptive attitudes, and condom use in adolescent romantic relationships: A dyadic approach. Journal of Research on Adolescence, 25(1), 51-62. https://doi.org/10.1111/jora.12091

Yimer, M., Abera, B., Mulu, W., \& Bezabih, B. (2013). Knowledge, attitude and practices of high risk populations on louse- borne relapsing fever in Bahir Dar city, north-west Ethiopia. Science Journal of Public Health, 2(1), 15-22. https://doi.org/10.11648/j.sjph.20140201.13

\section{Copyrights}

Copyright for this article is retained by the author(s), with first publication rights granted to the journal.

This is an open-access article distributed under the terms and conditions of the Creative Commons Attribution license (http://creativecommons.org/licenses/by/4.0/). 